\title{
Plant Growth Promoting Traits Exhibited by Metal Tolerant Bacterial Isolates of Industrial Effluent
}

\author{
Priti Binita Lakra* and Bibhuti Bhushan Mishra
}

Department of Microbiology, College of Basic Science and Humanities, Orissa University of Agriculture and Technology, Bhubaneswar, Odisha, India

*Corresponding author

\section{A B S T R A C T}

\begin{tabular}{|l|}
\hline Ke y w o r d s \\
$\begin{array}{l}\text { Industrial effluent, Heavy } \\
\text { metal, Antibiotic } \\
\text { resistance, PGP } \\
\text { characteristics }\end{array}$ \\
\hline Article Info \\
\hline $\begin{array}{l}\text { Accepted: } \\
\text { 22 April } 2018 \\
\text { Available Online: } \\
\text { 10 May } 2018\end{array}$ \\
\hline
\end{tabular}

\section{Introduction}

Population explosion coupled with rapid industrialization, urbanization and application of synthetic chemicals in agriculture have created a new order of by product causing environmental pollution by generating innumerable quantity of solid and liquid wastes. Such wastes are often loaded with heavy metal that has emerged as a major factor for reducing plant growth and agricultural productivity worldwide (Ma et al., 2016).

Bacteria have the adaptive capacity to develop resistance/tolerance towards metal and antibiotic due to constant exposure in the environments (Sarma et al., 2010). In these environments the activities like biosorption, bioprecipitation, extracellular sequestration, transport mechanisms, and/or chelation are developed by the microorganism and such resistance mechanisms are the basis for the use of microbes in bioremediation (Haferburg et al., 2007).

Plant growth promoting rhizobacteria (PGPR) are group of microorganism which facilitate plant growth under stress condition including toxic metal different direct and indirect traits and also helps bioremediation of toxic metals (Nadeem et al., 2014). Plant Growth Promoting Bacteria (PGPB) not only enhances plant growth but also increase productivity. 
They can produce phytohormones (e.g. indole3-acetic acid) that promote plant growth, facilitate nutrient availability (e.g. by solubilizing phosphorus and producing ammonia) can alter metal flow dynamics via siderophore release etc. PGPB can also produce 1-aminocyclopropane-1carboxylate (ACC) deaminase, which alleviates plant stress through the reduction of ethylene levels (Glick, 2012). A bacterial isolate with metal tolerant efficiency and plant growth promoting (PGP) properties can provide multiple advantages in crop productivity (Tripathy et al., 2007).

Keeping in view of this an attempt has been taken in the present study to assess the ability of bacteria isolated from effluent of a steel plant on metal tolerance and plant growth promoting traits.

\section{Materials and Methods}

\section{Collection of Sample}

Effluent sample was collected in a sterile bottle from industrial effluent from a steel plant aseptically and transferred to the laboratory and stored under refrigerated condition for further work.

\section{Enrichment, isolation and screening of bacterial isolates}

Effluent enrichment method was performed by placing $10 \mathrm{ml}$ effluent sample in a $250 \mathrm{ml}$ Erlenmeyer flask containing $100 \mathrm{ml}$ sterilized luria bertani media and incubated at $37^{\circ} \mathrm{C}$. After 48 hours of incubation, the turbid broth was spreaded on sterilized Luria bertani agar plate and incubated for $24 \mathrm{hrs}$.

The isolates were screened on LB agar plate amended with 50 ppm of heavy metals such as $\mathrm{Ni}, \mathrm{Cd}, \mathrm{Cr}, \mathrm{Pb}$ and $\mathrm{Hg}$ separately and incubated for 48 hours. The growth of heavy metal resistant bacteria colony having different morphology were observed and Purity of the isolates were confirmed by quadrant streak plate method and preserved using $15 \%$ glycerol stocks.

\section{Biochemical characterization and sugar utilization test}

Morphological Colony characteristics of the isolates were noted and grams staining were performed. Biochemical tests such as indole, methyl red, voges proskauer, citrate utilization test, oxidase and catalase test, $\mathrm{H}_{2} \mathrm{~S}$ production, motility test, and Urease production were carried out following standard method. Sugar utilisation was tested using Hicarbo Kit-A, Hicarbo Kit-B and Hicarbo Kit-C (Himedia).

\section{Assessment of metal tolerance}

Evaluation of all the bacterial isolate was determined by gradual increase of $\mathrm{Cr}, \mathrm{Ni}, \mathrm{Cd}$, $\mathrm{Pb}$ and $\mathrm{Hg}$ metal concentration in LB media until the bacterial isolates failed to grow on plates over 7 days of incubation. The MIC of respective isolates was determined.

\section{Determination of antibiotic sensitivity test}

Antibiotic sensitivity pattern of the isolates were tested by Kirby-Bauer disc diffusion method. After incubation the resistance and sensitivity were determined according to the inhibition zone around the colony.

\section{PGPB traits}

The metal tolerant bacterial isolate were analyzed for different plant growth promoting traits.

\section{Indole acetic acid producing ability}

Bacteria were incubated for $24 \mathrm{~h}$ in LB broth and then they were centrifuged. An aliquot of 
$1 \mathrm{ml}$ of supernatant was taken and transferred to a test tube that contained $2 \mathrm{ml}$ of Salkowski reagent. The mixture was kept in room temperature for $30 \mathrm{~min}$ (Gordon and Weber, 1951) to observe change on colouration.

\section{Solubilization of inorganic phosphate}

For phosphate solubilization assay, fresh cultures of bacterial strains were inoculated into National Botanical Research Institute Phosphate Medium (NBRIP) (Nautiyal, 1999). Plates were incubated for 7 days and the formed with clear zone around them were considered as positive for the phosphate solubilization.

\section{Ammonia production}

Bacterial strains were checked for ammonia production according to Cappuccino and Sherman (1992). Fresh cultures were inoculated in to $10 \mathrm{ml}$ peptone water. The production of ammonia was detected by adding $500 \mu \mathrm{l}$ of Nessler's reagent to each tube.

\section{Siderophore production}

Detection of Siderophore production was carried out by inoculating the bacterial isolates on Chrome Azurol S (CAS) plate assays (Schwyn and Neilands, 1987).

\section{HCN production}

The HCN production by the bacterial isolates was determined by adapting the method of Ahmad et al., (2008). Fresh cultures were inoculated in LB medium supplemented with glycin $(4.4 \mathrm{~g} / \mathrm{lit})$ for $24 \mathrm{~h}$ at $30^{\circ} \mathrm{C}$. On the top of each plate, a sterilized filter paper (Whatman no.1) soaked in 2\% sodium carbonate prepared in $0.5 \%$ picric acid solution was placed. Plates were incubated at $30^{\circ} \mathrm{C}$ to study $\mathrm{HCN}$ production.

\section{Results and Discussion}

Enrichment, isolation and screening of Bacterial isolates

A total of sixty bacterial isolates (R-1 to R-60) were isolated from effluent sample. Amongst them twenty bacterial isolates were found to be tolerant to $\mathrm{Ni}, \mathrm{Cr}, \mathrm{Cd}, \mathrm{Pb}$, and $\mathrm{Hg}$. Out of these twenty isolates, six bacterial isolates were gram negative and fourteen were gram positive. All the 20 isolates exhibited tolerance against all the five heavy metals up to a minimum concentration of $50 \mathrm{ppm}$ (Table $1)$.

\section{Biochemical characterization and sugar utilization test}

On biochemical variability and sugar utilization, it was observed that among 20 isolates only R-14 was positive for indole production and rest isolates were negative. Ten isolates were positive for methyl red and ten showed negative result.

Eight isolates showed positive for Voges Proskauer reaction and twelve were negative for the test. Twelve of the twenty bacterial isolates utilized citrate. Fourteen bacteria were positive for oxidase, seventeen were positive for catalase and nine isolates could produce hydrogen sulphide.

Among twenty, fifteen isolates were found to motile and thirteen isolates had the ability to produce urease enzyme. Total 35 numbers of sugars were taken for test. The results are described in table 2. 3 and 4. According to above biochemical reaction the isolates were identified by ABIS online are in table 5 .

\section{Tolerance for heavy metal concentrations (in ppm)}

All the 20 bacterial isolates isolated from industrial effluent were found to be tolerant to 
$\mathrm{Ni}, \mathrm{Cr}, \mathrm{Cd}, \mathrm{Pb}$ and $\mathrm{Hg}$. Isolates $\mathrm{R}-3, \mathrm{R}-4, \mathrm{R}-$ 10, R-22, R-26, R-35, R-43, R-44, showed the highest tolerance of $1350 \mathrm{ppm}$ to the metal $\mathrm{Ni}$ (Fig. 1). Isolate R-37 was able to tolerate $\mathrm{Cr}$ metal up to the concentration of $1650 \mathrm{ppm}$ (Fig. 2).

Most of the bacteria were tolerant to $120 \mathrm{ppm}$ of $\mathrm{Cd}$ concentration (Fig. 3). For $\mathrm{Pb}$ all the organisms were found to be tolerant at the concentrations of $75 \mathrm{ppm}$. Most of the isolates were tolerant to $50 \mathrm{ppm}$ concentration and both R-37 and R-56 were tolerant to 100 ppm of $\mathrm{Hg}$ (Fig. 4 and 5).

\section{Antibiotic susceptibility test}

Table 6 depicts that all the isolates resisted to all most all the commonly used antibiotics taken for this study. All the isolates are susceptible for penicillin B. All the bacteria were resistant to nalidixic acid, azithromycin, streptomycin, ciprofloxacin, kanamycin, and amoxycilin. A few organisms were susceptible for rifampicin, gentamycin, amikacin, cefonicid and tetracycline. Sarma et al., 2010 opined that the resistance capacity may develop due to the metal contaminated environment in isolates.

Table.1 Grams staining and morphological characteristics of the isolates

\begin{tabular}{|l|l|l|}
\hline Isolates & $\begin{array}{l}\text { Gram's } \\
\text { reaction }\end{array}$ & Morphological character \\
\hline $\mathbf{R}-3$ & +ve cocci & Creamy, cerrated, dry round ring at the centre, sorounded by layers \\
\hline $\mathbf{R}-4$ & + ve cocci & Cerrated margin, ring at centre \\
\hline $\mathbf{R}-\mathbf{1 0}$ & + ve rod & Small, round, Creamy, shiny, centre elevated and dry \\
\hline $\mathbf{R}-\mathbf{1 4}$ & - ve rod & Small, round, greenish brown, elevated shiny \\
\hline $\mathbf{R}-22$ & + ve cocci & Creamy, flat, dry, surrounded by wet margin. \\
\hline $\mathbf{R}-26$ & + ve rod & White, flat surrounded by dry margin \\
\hline $\mathbf{R}-35$ & + ve cocci & Small, creamy, dry, round, flat \\
\hline $\mathbf{R}-37$ & - ve rod & Light green, spreaded colony \\
\hline $\mathbf{R}-\mathbf{4 3}$ & - ve rod & Flat, cerrated margin \\
\hline $\mathbf{R}-44$ & + ve cocci & Mucoid colony, folded, wrinkled \\
\hline $\mathbf{R}-45$ & + ve cocci & White, cerrated margin, ring at centre \\
\hline $\mathbf{R}-51$ & + ve rod & Small, white, round \\
\hline $\mathbf{R}-52$ & + ve cocci & Small, Yellow, round \\
\hline $\mathbf{R}-53$ & + ve rod & Mucoid, cerrated margin \\
\hline $\mathbf{R}-54$ & + ve cocci & Mucoid, elevated colony, \\
\hline $\mathbf{R}-55$ & + ve rod & Small, round, white, elevated colony \\
\hline $\mathbf{R}-56$ & - ve rod & Small, round, watery, cerrated margin \\
\hline $\mathbf{R}-57$ & - ve rod & Lemon green, oily, spreaded \\
\hline $\mathbf{R}-58$ & - ve rod & Green, and mucoid colony \\
\hline $\mathbf{R}-60$ & + ve cocci & Round, offwhite \\
\hline
\end{tabular}


Int.J.Curr.Microbiol.App.Sci (2018) 7(5): 3458-3471

Table.2 Biochemical characterization of the isolates

\begin{tabular}{|c|c|c|c|c|c|c|c|c|c|}
\hline Isolates & $\begin{array}{l}\text { Indole } \\
\text { Test }\end{array}$ & $\begin{array}{l}\text { Methyl } \\
\text { red }\end{array}$ & $\begin{array}{l}\text { Voges } \\
\text { Proskauer }\end{array}$ & $\begin{array}{l}\text { Citrate } \\
\text { utilization }\end{array}$ & Oxidase & Catalase & $\begin{array}{l}\mathrm{H}_{2} \mathrm{~S} \\
\text { production }\end{array}$ & Motility & $\begin{array}{l}\text { Urea } \\
\text { hydrolysis }\end{array}$ \\
\hline R-3 & - & + & + & - & + & - & - & - & + \\
\hline $\mathbf{R}-4$ & - & + & + & + & - & + & + & + & - \\
\hline R-10 & - & + & + & + & - & + & + & + & - \\
\hline R-14 & + & + & - & + & + & + & - & + & - \\
\hline R-22 & - & + & + & + & - & + & + & - & + \\
\hline R-26 & - & + & - & - & - & - & + & + & + \\
\hline R-35 & - & + & - & + & + & + & + & + & + \\
\hline R-37 & - & - & - & + & + & + & - & + & - \\
\hline $\mathbf{R}-43$ & - & + & + & - & + & - & - & + & - \\
\hline$R-44$ & - & + & + & + & - & + & - & + & + \\
\hline $\mathbf{R}-45$ & - & + & + & + & + & + & + & - & + \\
\hline $\mathbf{R}-51$ & - & - & - & - & + & + & + & + & + \\
\hline R-52 & - & + & - & - & + & + & - & + & + \\
\hline R-53 & - & - & + & - & + & + & - & - & + \\
\hline $\mathbf{R}-54$ & - & - & - & - & + & + & + & + & + \\
\hline $\mathbf{R}-55$ & - & + & + & - & + & + & - & + & + \\
\hline $\mathbf{R}-56$ & - & - & - & - & - & + & - & + & + \\
\hline $\mathbf{R - 5 7}$ & - & - & - & + & + & + & - & + & - \\
\hline R-58 & - & - & - & + & + & + & - & + & - \\
\hline R-60 & - & - & - & + & + & + & + & - & + \\
\hline
\end{tabular}

*Legend: + (positive) reaction, - (negative) reaction. 


\section{Int.J.Curr.Microbiol.App.Sci (2018) 7(5): 3458-3471}

Table.3 Sugar fermentation of the isolates

\begin{tabular}{|c|c|c|c|c|c|c|c|c|c|c|c|c|c|c|c|c|c|c|}
\hline Isolates & lac & Xyl & Mal & Fruc & Dext & Gal & Raf & Tre & Mel & Suc & L-ara & Man & Inu & N-agl & Glyc & Sal & Dulc & Ins \\
\hline R-3 & - & - & - & + & + & - & + & + & - & + & - & - & + & - & + & - & - & - \\
\hline$R-4$ & - & - & - & + & + & + & + & + & + & + & + & + & + & - & + & - & - & + \\
\hline R-10 & + & - & - & + & + & - & - & - & + & + & - & - & - & - & + & + & - & - \\
\hline R-14 & + & + & + & + & + & + & + & + & + & + & - & + & + & - & + & + & + & + \\
\hline R-22 & - & + & + & + & + & - & + & + & - & + & - & + & + & + & + & + & + & - \\
\hline R-26 & - & + & + & + & + & + & - & - & + & - & + & + & + & - & + & - & - & - \\
\hline R-35 & - & - & + & + & + & - & - & - & - & + & - & - & - & + & + & + & - & + \\
\hline R-37 & - & - & - & - & - & - & - & - & - & - & - & - & + & + & + & + & - & + \\
\hline $\mathrm{R}-\mathbf{4 3}$ & - & - & + & + & + & - & + & + & - & + & + & + & - & - & + & - & - & - \\
\hline $\mathrm{R}-44$ & + & + & + & - & + & - & - & + & - & - & - & - & - & - & - & + & + & - \\
\hline $\mathbf{R}-45$ & + & + & + & + & + & - & + & - & - & - & + & + & + & + & - & - & + & - \\
\hline R-51 & - & - & - & - & - & + & - & - & + & + & + & - & - & - & - & - & + & + \\
\hline R-52 & - & + & - & - & + & + & - & - & - & - & + & - & - & - & + & + & - & + \\
\hline $\mathbf{R}-\mathbf{5 3}$ & + & + & + & + & + & - & + & + & - & - & - & + & - & + & + & - & + & - \\
\hline$R-54$ & + & + & - & - & - & - & + & + & + & + & - & + & - & + & - & - & + & - \\
\hline $\mathbf{R}-55$ & - & - & - & - & - & - & + & - & + & - & + & - & + & + & - & + & - & + \\
\hline R-56 & + & + & - & - & - & + & - & - & - & + & + & + & - & + & + & + & - & - \\
\hline $\mathbf{R}-57$ & - & + & - & - & + & + & - & - & + & - & + & + & - & - & - & + & + & - \\
\hline R-58 & - & + & - & - & + & + & - & - & - & - & + & + & - & - & + & - & + & - \\
\hline R-60 & + & + & + & + & + & - & + & + & - & - & + & + & - & + & - & - & - & + \\
\hline
\end{tabular}

Legend: + (positive) reaction, - (negative) reaction.

Lac-Lactose, Xyl-Xylose, Mal-Maltose, Fruc-Fructose, Dext-Dextrose, Gal-Galactose, Raf-Raffinose, Tre-Trehalose, Mel-Mellibiose, Suc-Sucrose, L-ara-Larabinose, Man-Mannose, Inu-Inulin, N-agl-Sodium- gluconate, Gly-Glycerol, Sal-Salicin, Dul-Dulcitol, Ins- Inositol. 


\section{Int.J.Curr.Microbiol.App.Sci (2018) 7(5): 3458-3471}

Table.4 Sugar fermentation of the isolates

\begin{tabular}{|c|c|c|c|c|c|c|c|c|c|c|c|c|c|c|c|c|c|}
\hline Isolates & Sorb & Mant & Ado & Arab & Erythr & $a$-methyl & Rha & Celli & melz & $\alpha-m a n o$ & Xylt & ONPG & Esc & $\begin{array}{l}\text { D- } \\
\text { ara }\end{array}$ & Citr & Maln & Sorb \\
\hline $\mathbf{R}-\mathbf{3}$ & - & + & - & - & - & + & - & + & - & - & - & - & + & - & - & - & + \\
\hline $\mathbf{R}-4$ & + & + & - & - & + & - & - & + & - & - & + & - & + & - & + & - & - \\
\hline R-10 & - & + & - & + & + & - & - & + & + & - & + & + & + & + & + & - & - \\
\hline R-14 & + & + & - & - & - & - & + & - & - & - & - & + & + & + & + & - & - \\
\hline R-22 & + & + & + & - & - & - & + & + & - & - & - & - & + & + & + & - & - \\
\hline R-26 & + & + & + & - & - & - & + & - & - & - & - & - & + & + & - & - & - \\
\hline R-35 & + & - & - & + & - & - & + & - & - & - & + & - & + & - & + & + & - \\
\hline R-37 & + & + & - & - & + & - & + & - & - & - & - & - & + & - & + & - & - \\
\hline$R-43$ & - & - & - & - & - & - & - & + & - & - & - & - & + & - & - & - & - \\
\hline$R-44$ & - & + & - & - & + & - & - & - & - & - & - & - & + & - & + & - & - \\
\hline$R-45$ & - & + & - & + & - & - & - & + & - & - & + & - & + & - & + & - & - \\
\hline $\mathrm{R}-51$ & + & + & + & - & - & - & + & + & - & - & + & - & + & - & + & - & - \\
\hline R-52 & + & - & + & - & - & + & + & - & - & - & - & - & + & - & + & - & - \\
\hline R-53 & + & + & + & - & + & + & - & + & - & - & + & - & + & - & + & - & - \\
\hline R-54 & + & - & + & + & - & + & - & + & - & - & - & - & + & - & + & - & - \\
\hline R-55 & - & + & - & + & - & + & + & - & - & - & + & - & + & - & - & + & - \\
\hline R-56 & + & - & + & + & - & - & + & + & - & - & + & - & + & - & - & - & - \\
\hline R-57 & + & - & - & + & + & - & - & - & - & - & + & - & + & - & + & + & - \\
\hline R-58 & - & - & - & - & - & + & - & - & - & + & + & + & + & - & + & + & - \\
\hline R-60 & - & + & + & - & - & - & + & + & - & + & + & - & + & - & + & + & - \\
\hline
\end{tabular}

Legend: + (positive) reaction, - (negative) reaction.

Sorb- Sorbitol, Mant- Mannitol, Ado- Adonitol, Arab- Arabitol, Erythr- Erythritol, $\alpha$-methyl- alpha-Methyl-D-glucoside, Rha-Rhamnose, Celli- Cellobiose, melz- Melezitose, $\alpha$-mano- alpha-Methyl-D-Mannoside, Xylt-Xylitol, ONPG-ortho-Nitrophenyl- $\beta$-galactoside, Esc-Esculin, D-ara- D-Arabinose, Citr- Citrate, Maln- Malonate, Sorb- Sorbose. 
Table.5 Biochemical identification of the isolates

\begin{tabular}{|l|l|}
\hline Isolates & Identification \\
\hline $\mathbf{R}-3$ & Staphylococcus $s p$. \\
\hline $\mathbf{R}-4$ & Staphylococcus $s p$. \\
\hline $\mathbf{R}-10$ & Bacillus $s p$. \\
\hline $\mathbf{R}-14$ & Burkholderia $s p$. \\
\hline $\mathbf{R}-22$ & Staphylococcus $s p$. \\
\hline $\mathbf{R}-26$ & Bacillus sp. \\
\hline $\mathbf{R}-35$ & Staphylococcus $s p$. \\
\hline $\mathbf{R}-37$ & Unknown taxon \\
\hline $\mathbf{R}-43$ & Sphingomonas $s p$. \\
\hline $\mathbf{R}-44$ & Staphylococcus $s p$. \\
\hline $\mathbf{R}-45$ & Unknown taxon \\
\hline $\mathbf{R}-51$ & Paenibacillus $s p$. \\
\hline $\mathbf{R}-52$ & Staphylococcus $s p$. \\
\hline $\mathbf{R}-53$ & Paenibacillus $s p$. \\
\hline $\mathbf{R}-54$ & Unknown taxon \\
\hline $\mathbf{R}-55$ & Lysinibacillus $s p$. \\
\hline $\mathbf{R}-56$ & Unknown taxon \\
\hline $\mathbf{R}-57$ & Pseudomonas $s p$. \\
\hline $\mathbf{R}-58$ & Pseudomonas $s p$. \\
\hline $\mathbf{R}-60$ & Enterococcus $s p$. \\
\hline
\end{tabular}


Table.6 Antibiotic susceptibility of the metal tolerant bacterial isolates

\begin{tabular}{|c|c|c|c|c|c|c|c|c|c|c|c|c|c|}
\hline Isolates & PEN & RIF & NA & $\mathbf{A Z M}$ & STR & GEN & CIP & $\mathbf{K A N}$ & $\mathbf{A M C}$ & CID & TET & NV & AMX \\
\hline R-3 & + & - & - & - & - & - & - & - & - & - & - & - & - \\
\hline $\mathbf{R}-4$ & + & - & - & - & - & - & - & - & - & - & + & - & - \\
\hline R-10 & + & - & - & - & - & - & - & - & - & - & - & - & - \\
\hline R-14 & + & - & - & - & - & + & - & - & - & + & + & + & - \\
\hline R-22 & + & - & - & - & - & - & - & - & + & - & + & - & - \\
\hline$R-26$ & + & - & - & - & - & - & - & - & + & - & - & - & - \\
\hline R-35 & + & - & - & - & - & - & - & - & - & + & - & - & - \\
\hline R-37 & + & + & - & - & - & - & - & - & - & - & + & - & - \\
\hline$R-43$ & + & - & - & - & - & - & - & - & - & - & - & - & - \\
\hline$R-44$ & + & - & - & - & - & + & - & - & - & - & - & - & - \\
\hline$R-45$ & + & - & - & - & - & - & - & - & - & - & + & - & - \\
\hline $\mathbf{R}-51$ & + & - & - & - & - & - & - & - & - & - & - & - & - \\
\hline R-52 & + & - & - & - & - & - & - & - & - & - & - & - & - \\
\hline R-53 & + & - & - & - & - & - & - & - & - & - & - & - & - \\
\hline R-54 & + & - & - & - & - & + & - & - & - & - & - & - & - \\
\hline R-55 & + & - & - & - & - & + & - & - & - & - & - & - & - \\
\hline R-56 & + & - & - & - & - & - & - & - & - & - & - & - & - \\
\hline $\mathbf{R}-57$ & + & - & - & - & - & - & - & - & - & - & - & - & - \\
\hline R-58 & + & - & - & - & + & - & - & - & - & - & + & - & - \\
\hline$R-60$ & + & - & + & - & + & - & - & - & - & + & - & - & - \\
\hline
\end{tabular}

Legend: + (Susceptible), - (Resistant)

PEN-penicillin G, RIF-Rifampicin, NA-Nalidixic acid, AZM-Azithromycin, STR-Streptomycin, GEN-Gentamycin, CIP-Ciprofloxacin, KAN-Kanamycin, AMC-Amikacin, CID-Cefonicid, TET-Tetracyclin, NV-Novobiocin, and AMX-Amoxycilin 
Table.7 Plant growth promotion traits of the metal tolerant bacterial isolates

\begin{tabular}{|c|c|c|c|c|c|}
\hline Isolates & IAA & $\begin{array}{l}\text { Phosphate } \\
\text { solubilization }\end{array}$ & $\begin{array}{l}\text { Ammonia } \\
\text { production }\end{array}$ & $\begin{array}{l}\text { Siderophore } \\
\text { production }\end{array}$ & $\begin{array}{l}\text { Hydrogen } \\
\text { Cyanide } \\
\text { production }\end{array}$ \\
\hline R-3 & $-\mathrm{Ve}$ & -ve & $+\mathrm{Ve}$ & $-\mathrm{Ve}$ & $-\mathrm{Ve}$ \\
\hline$\overline{R-4}$ & $-\mathrm{Ve}$ & -ve & $+\mathrm{Ve}$ & $-\mathrm{Ve}$ & $-\mathrm{Ve}$ \\
\hline R-10 & $-\mathrm{Ve}$ & -ve & $+\mathrm{Ve}$ & $-\mathrm{Ve}$ & $-\mathrm{Ve}$ \\
\hline$R-14$ & $+\mathrm{Ve}$ & -ve & $+\mathrm{Ve}$ & $+\mathrm{Ve}$ & $-\mathrm{Ve}$ \\
\hline R-22 & $-\mathrm{Ve}$ & -ve & $-\mathrm{Ve}$ & $-\mathrm{Ve}$ & $-\mathrm{Ve}$ \\
\hline R-26 & $-\mathrm{Ve}$ & -ve & $-\mathrm{Ve}$ & $-\mathrm{Ve}$ & $-\mathrm{Ve}$ \\
\hline R-35 & $-\mathrm{Ve}$ & -ve & $+\mathrm{Ve}$ & $+\mathrm{Ve}$ & $-\mathrm{Ve}$ \\
\hline R-37 & $+\mathrm{Ve}$ & -ve & $+\mathrm{Ve}$ & $+\mathrm{Ve}$ & $-\mathrm{Ve}$ \\
\hline$R-43$ & $-\mathrm{Ve}$ & -ve & $+\mathrm{Ve}$ & $-\mathrm{Ve}$ & $-\mathrm{Ve}$ \\
\hline$R-44$ & $-\mathrm{Ve}$ & -ve & $+\mathrm{Ve}$ & $-\mathrm{Ve}$ & $-\mathrm{Ve}$ \\
\hline$R-45$ & $-\mathrm{Ve}$ & -ve & $+\mathrm{Ve}$ & $-\mathrm{Ve}$ & -Ve \\
\hline R-51 & $-\mathrm{Ve}$ & -ve & $+\mathrm{Ve}$ & $-\mathrm{Ve}$ & $-\mathrm{Ve}$ \\
\hline R-52 & $-\mathrm{Ve}$ & -ve & $-\mathrm{Ve}$ & $-\mathrm{Ve}$ & $-\mathrm{Ve}$ \\
\hline R-53 & $-\mathrm{Ve}$ & -ve & $-\mathrm{Ve}$ & $-\mathrm{Ve}$ & $-\mathrm{Ve}$ \\
\hline R-54 & $-\mathrm{Ve}$ & -ve & $+\mathrm{Ve}$ & $-\mathrm{Ve}$ & $-\mathrm{Ve}$ \\
\hline R-55 & $-\mathrm{Ve}$ & -ve & $-\mathrm{Ve}$ & $-\mathrm{Ve}$ & $-\mathrm{Ve}$ \\
\hline R-56 & $+\mathrm{Ve}$ & -ve & $+\mathrm{Ve}$ & $+\mathrm{Ve}$ & $-\mathrm{Ve}$ \\
\hline $\mathbf{R}-57$ & $+\mathrm{Ve}$ & $+v e$ & $+\mathrm{Ve}$ & $+\mathrm{Ve}$ & $+\mathrm{Ve}$ \\
\hline R-58 & $+\mathrm{Ve}$ & $+\mathrm{ve}$ & $+\mathrm{Ve}$ & $+\mathrm{Ve}$ & $+\mathrm{Ve}$ \\
\hline R-60 & $-\mathrm{Ve}$ & -ve & $+\mathrm{Ve}$ & $-\mathrm{Ve}$ & $-\mathrm{Ve}$ \\
\hline
\end{tabular}

Fig.1 Growth of 20 isolates at different concentrations of Ni metal

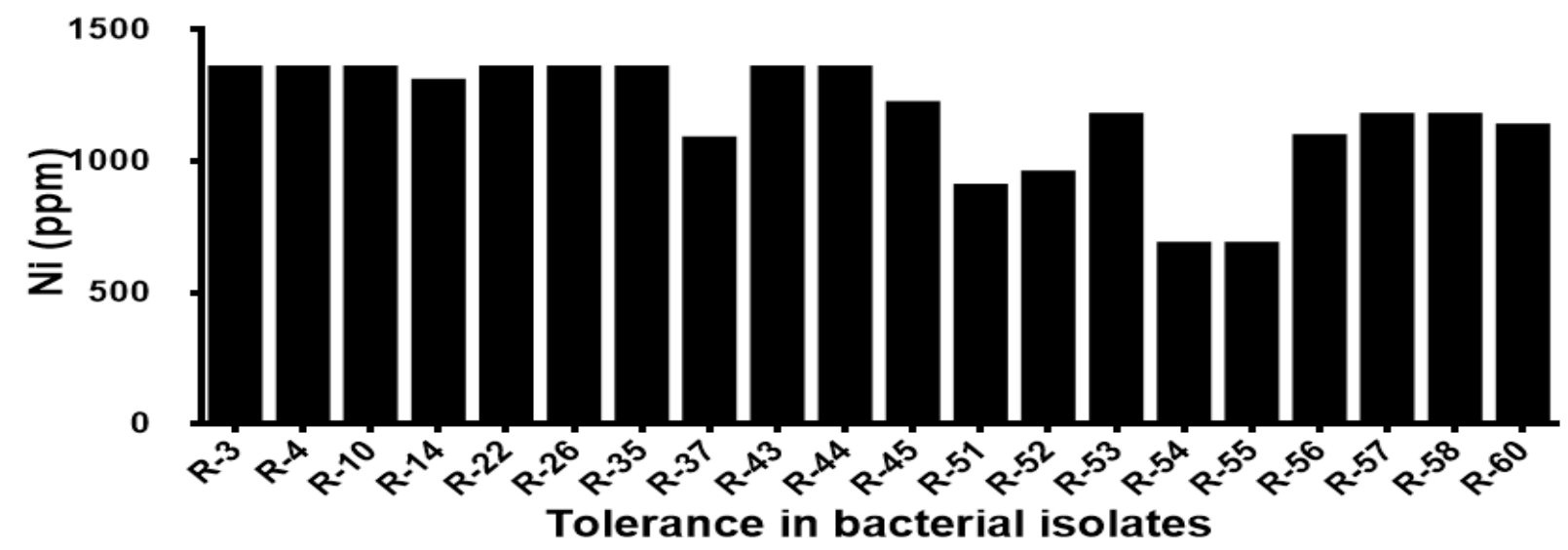


Fig.2 Growth of 20 isolates at different concentrations of $\mathrm{Cr}$ metal

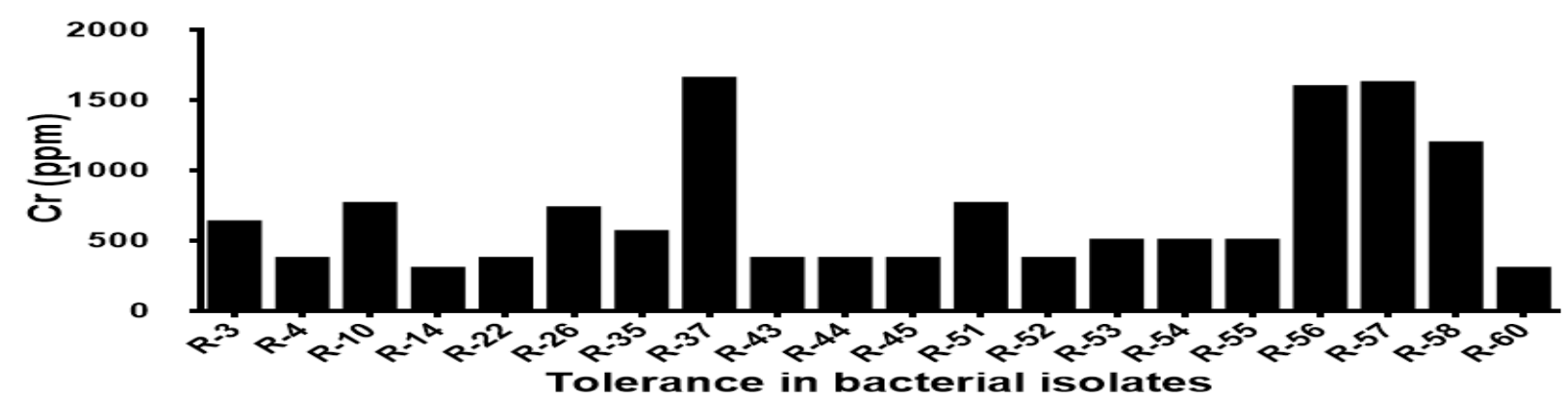

Fig.3 Growth of 20 isolates at different concentrations of $\mathrm{Cd}$ metal

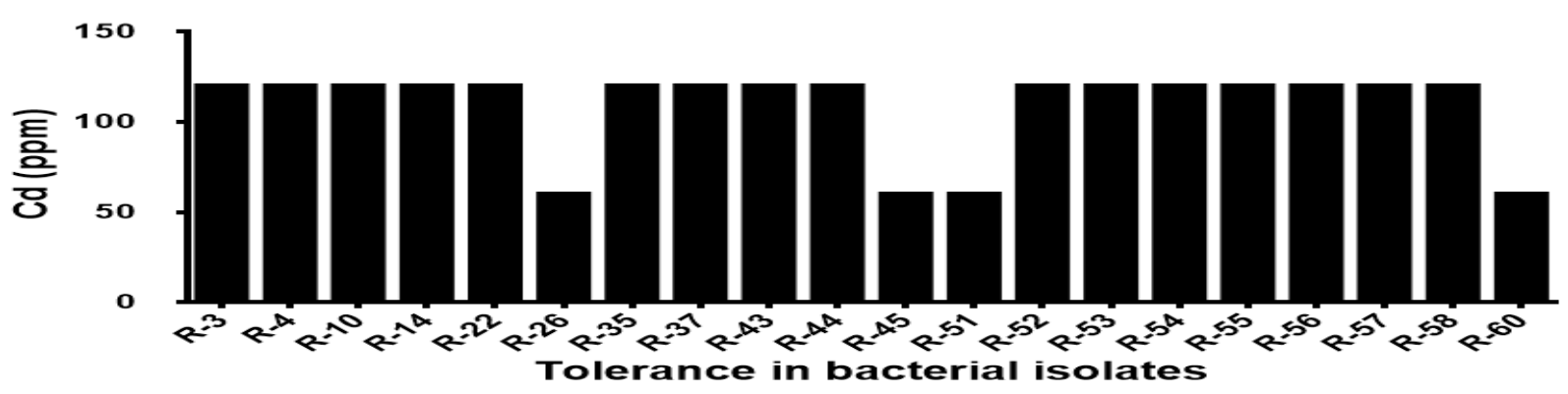

Fig.4 Growth of 20 isolates at different concentrations of $\mathrm{Pb}$ metal

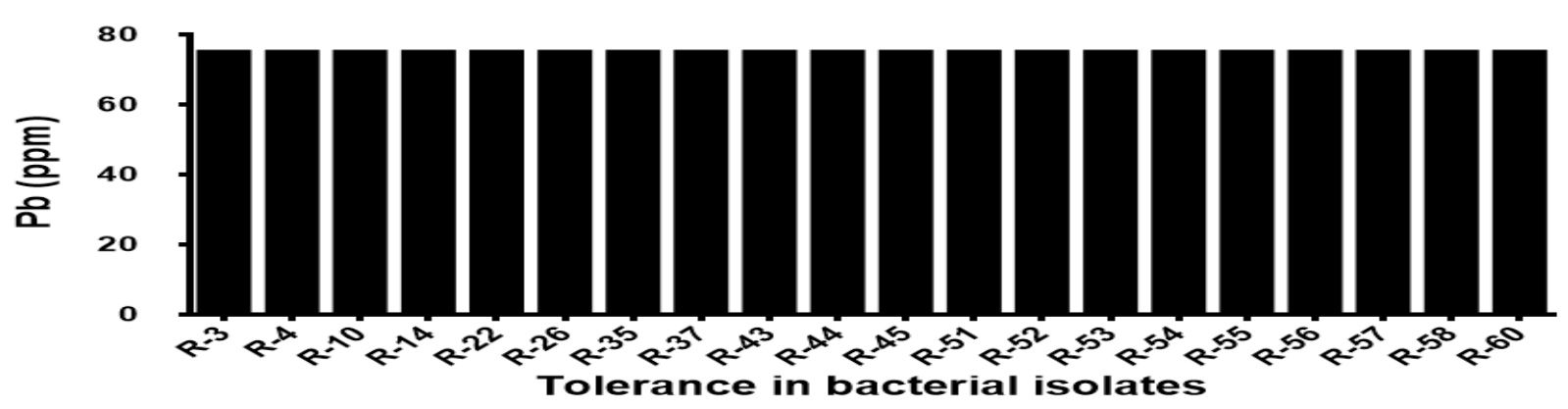

Fig.5 Growth of 20 isolates at different concentrations of $\mathrm{Hg}$ metal

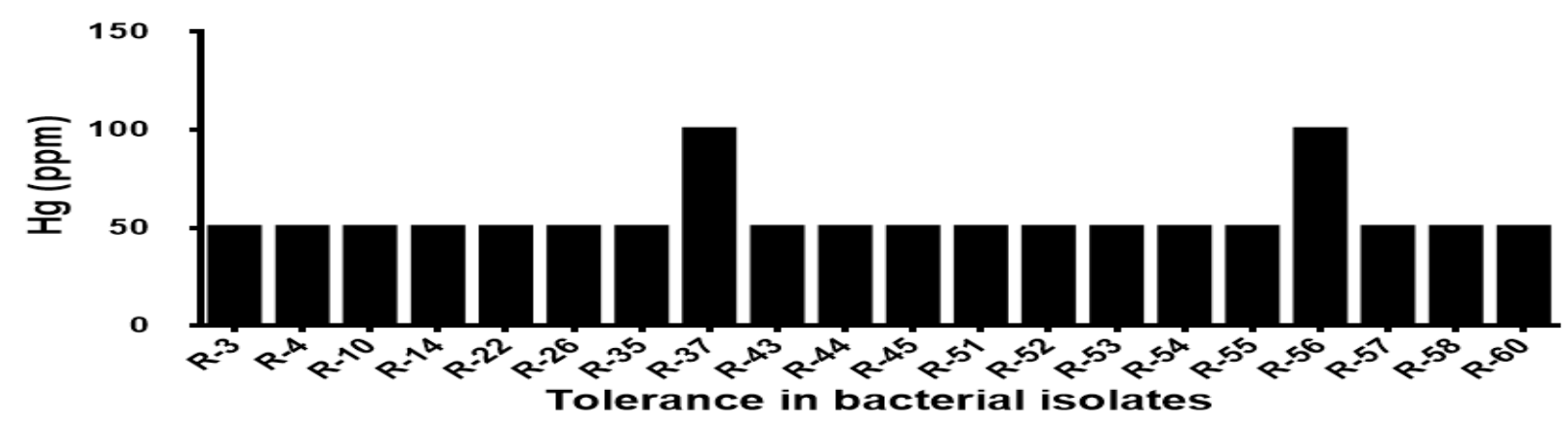




\section{Plant growth promotion activities}

Observation of pink colour in IAA tube indicates positive result. R-14, R-37 and R$56, \mathrm{R}-57$ and R-58 were found to be positive for IAA production. NBRIP Plates were incubated for 7 days and the formed with clear zone around the colony were considered as positive for the phosphate solubilization (Table 7). R-57 and R-58 showed positive result for $\mathrm{PO}_{4}$ solubilisation. After adding Nessler's reagent in the ammonia tube, the development of yellow to brown colour was considered as positive result for ammonia production. Isolates R-22, R-26, R-52, R-53 and $\mathrm{R}-55$ showed positive result for ammonia production. The presence of yellow to orange halo around the bacterial growth after incubation for $72 \mathrm{~h}$ indicated a positive result for siderophore production. R-14, R-35, R-37, $\mathrm{R}-56, \mathrm{R}-57$ and $\mathrm{R}-58$ were found to be positive for siderophore. Change in colour of filter paper to orange colour was considered as positive result for $\mathrm{HCN}$ production. Both the isolates R-57 and R-58 were found to be positive for $\mathrm{HCN}$ production.

In the present investigation it is observed that all 20 bacterial isolates isolated from the industrial effluent were biochemically identified as Staphylococcus spp., Psuedomonas spp., Burkholderia sp., Sphingomonas sp., Bacillus spp., Paenibacillus sp., Lysinibacillus $s p$. and Enterococcus sp., and were found tolerant to 60-120ppm of $\mathrm{Cd}, 75 \mathrm{ppm} \mathrm{Pb}$ and 50-100 ppm $\mathrm{Hg}$ metals. These microorganisms have high degree of tolerance capacity against chromium ranging from $300-1650 \mathrm{ppm}$ and for nickel 680-1350ppm. Pandit et al., (2013) isolated six number of $\mathrm{Cu}, \mathrm{Cd}, \mathrm{Cr}$ and $\mathrm{Ni}$ tolerant bacteria from industrial effluent sample. So in this study it is evident that most of the bacterial isolates were found to be tolerant to the metal salts such as $\mathrm{Ni}, \mathrm{Cr}, \mathrm{Cd}$, $\mathrm{Pb}$ and $\mathrm{Hg}$ with high concentrations.
Pramanik et al., (2016) reported the chromium tolerant bacteria showing metal tolerant along with plant growth promotion (PGP) traits which might be helpful for plant growth promotion in chromium contaminated soil.

The results obtained in this study revealed that multiple heavy metal tolerant isolates are resistant to several antibiotics such as nalidixic acid, azithromycin, streptomycin, ciprofloxacin, kanamycin, and amoxycilin, rifampicin, gentamycin, amikacin, cefonicid and tetracycline. All the isolates are resistant to several antibiotics taken in this study except penicillin B. Kaur et al., (2015) reported that bacteria isolated from industrial effluents were found to be resistant against ampicillin, ciprofloxacin, erythromycin, kanamycin, tetracycline, vancomycin, methicillin, gentamycin, and chloramphenicol. Due to cross resistance mechanism the bacteria have tolerance more than one antimicrobial agent such as antibiotics and heavy metals (Champan, 2003). These were seemed to be an efficient plant growth promoting bacteria that could produce IAA, siderophore, ammonia, HCN and solubilize phosphate. Reports available also indicate that the potential of soil microorganism to assist plant establishment on contaminated environment through mediating nutrient mineralization and uptake by plants, production of plant growth hormones and siderophores. The beneficial effects caused by the indigenous isolates may be used as a bioinoculant that facilitates the plant growth and thus increases phytoextraction efficiency for the remediation of heavy metal contaminated sites. Psuedomonas sp. Showed potentials in metal absorption as well as PGP and biocontrol activity (Sarma et al., 2012).

The potential of the isolates can be considered as a probable candidate for the concerted 
approach in the bioremediation as well as phytoremediation technology to convert metal contaminated sites to productive land and increase crop prodictivity.

\section{Acknowledgement}

We are grateful to the Department of Microbiology, College of Basic Science and Humanities, Orissa University of Agriculture and Technology. Bhubaneswar, Odisha, India for the laboratory facility.

\section{References}

Ahmad, f., Ahmad, I., Khan, M. S., 2008. Screening of free-living rhizosphere bacteria for their multiple plant growth promoting activities. Microbiol. Res. 163(2), 173-181.

Antil, R. S., 2014. Problems and prospectus of utilization of sewage and industrial wastewaters in agriculture. Toxicological \& Environmental Chemistry, 96(8), 1260-1271.

Brahmachari, P.V., Rama, D., Reddy, S., Kotresha, D., 2016. Biodegradation of Catechol by free and immobilized cells of Achromobacter xylosoxidans strain 15DKVB isolated from paper and pulp industrial effluents. Biocatal. Agril. Biotechnol. 7, 36-44.

Cappuccino, J. C., Sherman, N., 1992. Microbiology: A laboratory Mannual. Third ed. Benjamin/Cummings Pub. co. New York. pp. 125-179.

Carlos, M. H. J., Stefani, P.V.Y., Janette, A. M., Melani, M. S. S., Gabriela, P. O., 2016. Assesing the effects of heavy metals in ACC deaminase and IAA production on plant growth-promoting bacteria. Microbiol. Res. 188-189, 5361.

Chapman, J. S. 2003. Disinfectant resistance mechanisms, cross-resistance, and co- resistance. Int. Biodeterior. Biodegradation. 51, 271-276.

Faisal, I., Tahira, Y., Muhammad, S. A., Muhammad, R., Sher, M. S., Qaiser, I., Irfan, A., 2016. Combined ability of Chromium $(\mathrm{Cr})$ tolerant plant growth promoting bacteria (PGPB) and salicylc acid (SA) in attenuation of chromium stress in maize plants. J. Plant. Physiol. and Biochem.108, 456-467.

Glick, B. R., 2012. Plant growth promoting bacteria: mechanisms and applications. Scientifica (Cairo) 15.

Gordon, S. A., weber, R. P., 1951. Colorimetric estimation of indole acetic acid. Plant. Physiol. 192-195.

Haferburg, G., Kothe, E., 2007. Microbes and metals: interactions in the environment. J. Basic. Microbiol. 47, 453-467.

Islam, F., Yasmeen, T., Arif, M. S., Riaz, M., Shahzad, S. M., Imran, Q., Ali, I., 2016.Combined ability of chromium $(\mathrm{Cr})$ tolerant plant growth promoting bacteria (PGPB) and salicylic $\operatorname{acid}(\mathrm{SA})$ in attenuation of chromium stress in maize plants. Plant. Physiol. Biochem.108, 456-467.

Kaur, S., Kaur, H. P., Rani, R., 2015 Isolation and characterization of heavy metal and antibiotic resistant bacteria from industrial effluent. World. J. Pharma. Pharmaceutical. Sci. 4(9), 765-772.

Lazar, V., Cernat, R., Balotescu, C., Cotar, A., Coipan, E., Cojocaru, C., 2002. Corelation between multiple antibiotic resistance and heavy-metal tolerance among some E.coli strains isolated from polluted waters. Bacteriol. Virusol. Parazitol. Epidemiol. 47(3-4), 155-60.

Ma, Y., Rajkumar, M., Zhang, C., Freitas, H., 2016. Beneficial role of bacterial endophytes in heavy metal phytoremediation. J. Environ. Manag. 174, 14-25.

Moreira, H., Pereira, S. I. A., Marques, A. P. G. C., Rangel., A. O. S. S., Castro, P. 
M. L., 2016. Selection of metal resistant plant growth promoting rhizobacteria for the growth and metal accumulation of energy in maize soil-Effect of inoculum size. Geoderma. 278, 1-11.

Nadeem, S.M., Ahmad, M., Zahir, Z. A., Javaid, A., Ashraf, M., 2014. The role of micorhyzae and plant growth promoting rhizobacteria (PGPR) in improving crop productivity under stressful environments. Biotechnol. Adv. 32, 429-448.

Nautiyal, C, S., 1999. An efficient microbiological growth medium for screening phosphate solubilizing microorganism. FEMS Microbiol. Lett.170, 265-270.

Pandit, R. J., Patel, B., Kunjadia, P. D., Nagee, A., 2013. Isolation characterization and molecular identification of heavy metal resistant bacteria from industrial effluents, Amala-Khadi-Ankleshwar, Gujurat. Int. J. Environ. Sci. 3, 5.

Pramanik, P., Ghosh, P. K., Ghosh, A., Sarkar, A., Maiti, T. K., 2016. Characterization of PGP Traits of a Hexavalent Chromium Resistant Raoultella $s p$. Isolated from the Rice Field near Industrial Sewage of Burdwan District, WB, India. Soil and Sediment Contamination. 25(3), 313331.
Rashmi, Y.C., R. Reshmi., R. Poornima., Kumar, S., 2017. Isolation and Characterization of Microorganisms from Agriculture Soil of Magnifera indica Orchard. Int. J. Curr. Microbiol. App. Sci. 6(6), 2707-2713.

Sarma B, Acharya C, Joshi SR 2010. Pseudomonads: a versatile bacterial group exhibiting dual resistance to metals and antibiotics. Afr. J. Microbiol. Res. 4, 2828-2835.

Sarma, B., Acharya, A. C., Santa, R., 2014..Plant growth promoting and metal bioadsorption activity of metal tolerant Pseudomonas aeruginosa isolate characterized from uranium ore deposit. Sect. Biol. Sci. 84(1), 157-164.

Schwyn, B., Neilands, J. B., 1987. Universal chemical assay for the detection and determination of siderophores. Anal. Biochem.160, 47-56.

Singh, Y., Ramteke, P.W., Tripathy, A., Shukla, P. K., 2013. Isolation and characterization of Bacillus resistant to multiple heavy metals. Int. J. Current. Microbiol. Appl. Sci. 2(11), 525-530.

Tripathi, P., Srivastava, S., 2007. Development and characterization of nickel accumulating mutants of Aspergillus nidulans. Indian J. Microbiol. 47, 241-250.

\section{How to cite this article:}

Priti Binita Lakra and Bibhuti Bhushan Mishra. 2018. Plant Growth Promoting Traits Exhibited by Metal Tolerant Bacterial Isolates of Industrial Effluent. Int.J.Curr.Microbiol.App.Sci. 7(05): 3458-3471. doi: https://doi.org/10.20546/ijcmas.2018.705.400 